\title{
Truncated Akash distribution: properties and applications
}

\begin{abstract}
In this paper, Truncated Akash distribution has been proposed. Its mean and variance have been derived. Nature of cumulative distribution and hazard rate functions have been derived and presented graphically. Its moments including Coefficient of Variation, Skenwness, Kurtosis and Index of dispersion have been derived. Maximum likelihood method of estimation has been used to estimate the parameter of proposed model. It has been applied on three data sets and compares its superiority over one parameter exponential, Lindley, Akash, Ishita and truncated Lindley distribution.
\end{abstract}

Keywords: Akash distribution, pranav distribution, maximum likelihood estimation, moments, hazard rate
Volume 9 Issue 5 - 2020

\author{
Kamlesh Kumar Shukla,' Rama Shanker² \\ 'Department of Statistics, Mainefhi College of Science, Eritrea \\ ${ }^{2}$ Department of Statistics, Assam University, Silcher, India
}

Correspondence: Kamlesh Kumar Shukla, Department of Statistics, Mainefhi College of Science, State of Eritrea, Emailkkshukla22@gmail.com

Received: August 7, 2020 | Published: October 26, 2020

\section{Introduction}

In the recent past decades, life time modeling has been becoming popular in distribution theory, where many statisticians are involved in introducing new models. Some of the life time models are very popular and applied in biological, engineering and agricultural areas, such as Lindley distribution of Lindley, ${ }^{1}$ weighted Lindley distribution introduced by Ghitany, Atieh, and Nadarajah, ${ }^{2}$ Akash distribution suggested by Shanker, ${ }^{3}$ Ishita distribution proposed by Shanker and Shukla, ${ }^{4}$ Pranav distribution introduced by Shukla, ${ }^{5}$ are some among others and extension of above mentioned distribution has also been becoming popular in different areas of statistics.

Shanker ${ }^{3}$ proposed Akash distribution convex combination of exponential and gamma distributions which is defined by its pdf and cdf

$$
\begin{array}{r}
f_{1}(y ; \theta)=\frac{\theta^{3}}{\theta^{2}+2}\left(1+y^{2}\right) e^{-\theta y} ; y>0, \theta>0 \\
F_{2}(y ; \theta)=1-\left[1+\frac{\theta y(\theta y+2)}{\theta^{2}+2}\right] e^{-\theta y} ; y>0, \theta>0
\end{array}
$$

The $r$ th moment about origin $\mu_{r}{ }^{\prime}$ of Akash distribution obtained by Shanker is

$$
\mu_{r}^{\prime}=\frac{r !\left\{\theta^{2}+(r+1)(r+2)\right\}}{\theta^{r}\left(\theta^{2}+2\right)} ; r=1,2,3, \ldots
$$

Shanker ${ }^{3}$ has discussed in details about its mathematical and statistical properties, estimation of parameters and applications to model lifetime data from engineering and biomedical engineering.

Truncated type of distribution are more effective for modeling life time data because its limits used as bound either upper or lower or both according to the given data. Truncated normal distribution is proposed by Johnson, Kotz, and Balakrishnan. ${ }^{6}$ It has wide application in economics and statistics. Many researchers have been proposed truncated type of distribution and applied in different areas of statistics, especially in censor data such as truncated Weibull distribution of Zange and Xie, ${ }^{12}$ truncated Lomax distribution of Aryuyuen and Bodhisuwan, ${ }^{8}$ truncated Pareto distribution of Janinetti and Ferraro, ${ }^{9}$ truncated Lindley distribution of Singh, Singh, and Sharma. ${ }^{10}$ Truncated version of a continuous distribution can be defined as:

Definition1. Let $\mathrm{X}$ be a random variable distributed according to some pdf $g(x ; \theta)$ and cdf $G(x ; \theta)$, where $\theta$ is a parameter vector of $\mathrm{X}$. Let $\mathrm{X}$ lies within the interval $[a, b]$, where $-\infty<a \leq x \leq b<\infty$, then $X$, conditional on $a \leq x \leq b$ is distributed as truncated distribution. The pdf of truncated distribution as reported by Singh, Singh, and Sharma ${ }^{10}$ defined by:

$$
f(x ; \theta)=g(x / a \leq x \leq b ; \theta)=\frac{g(x ; \theta)}{G(b ; \theta)-G(a ; \theta)}
$$

where $f(x ; \theta)=g(x ; \theta)$ for all $a \leq x \leq b$ and $f(x ; \theta)=0$ elsewhere.

Note that $f(x ; \theta)$ in fact is a pdf of X on interval $[a, b]$. We have

$$
\begin{gathered}
f(x ; \theta)=\int_{a}^{b} f(x ; \theta) d x=\frac{1}{G(b ; \theta)-G(a ; \theta)} \int_{a}^{b} g(x ; \theta) d x \\
=\frac{1}{G(b ; \theta)-G(a ; \theta)} G(b ; \theta)-G(a ; \theta)=1
\end{gathered}
$$

The cdf of truncated distribution is given by

$$
F(x ; \theta)=\int_{a}^{x} f(x ; \theta) d x=\frac{G(x ; \theta)-G(a ; \theta)}{G(b ; \theta)-G(a ; \theta)}
$$

The main objective of this paper is to propose new truncated distribution using Akash distribution, which is called as truncated Akash distribution. It has been divided in seven sections. Introduction about the paper is described in the first section. In the second section, truncated Akash distribution has been derived. Behavior of hazard rate has been presented in third section Statistical properties including its moment have been discussed in the fourth section.. Estimation of parameters of the proposed distribution has been discussed in the fifth section. Its application and comparative study with one parameter life time distribution have been illustrated in the section sixth. Finally the conclusion of the paper has been given in the seventh section. 

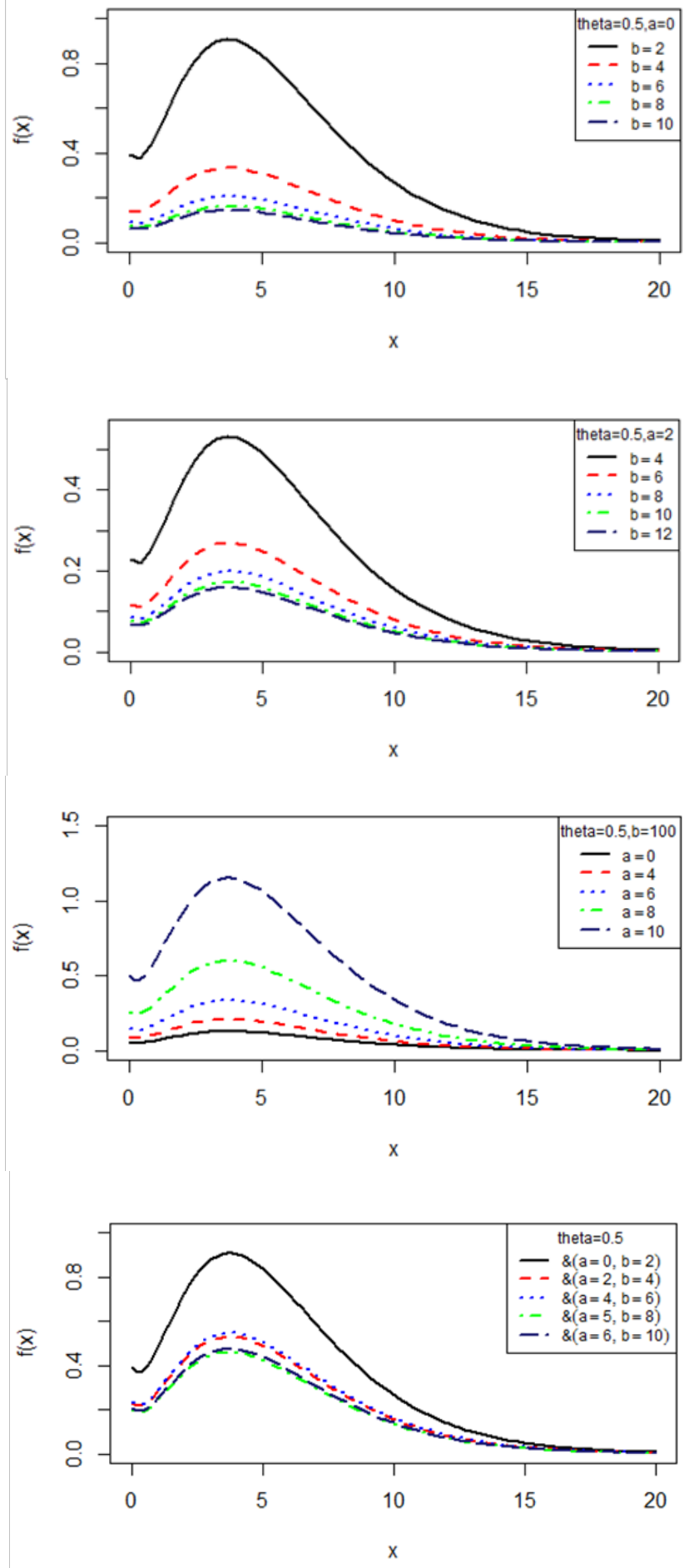

Figure I pdf plots of TAD for varying values of parameters.

\section{Truncated Akash distribution}

In this section, pdf and cdf of new truncated distribution is proposed and named Truncated Akash distribution, using (1.3) \& (1.4) of definition 1 and from (1.1) \& (1.2), which is defined as:
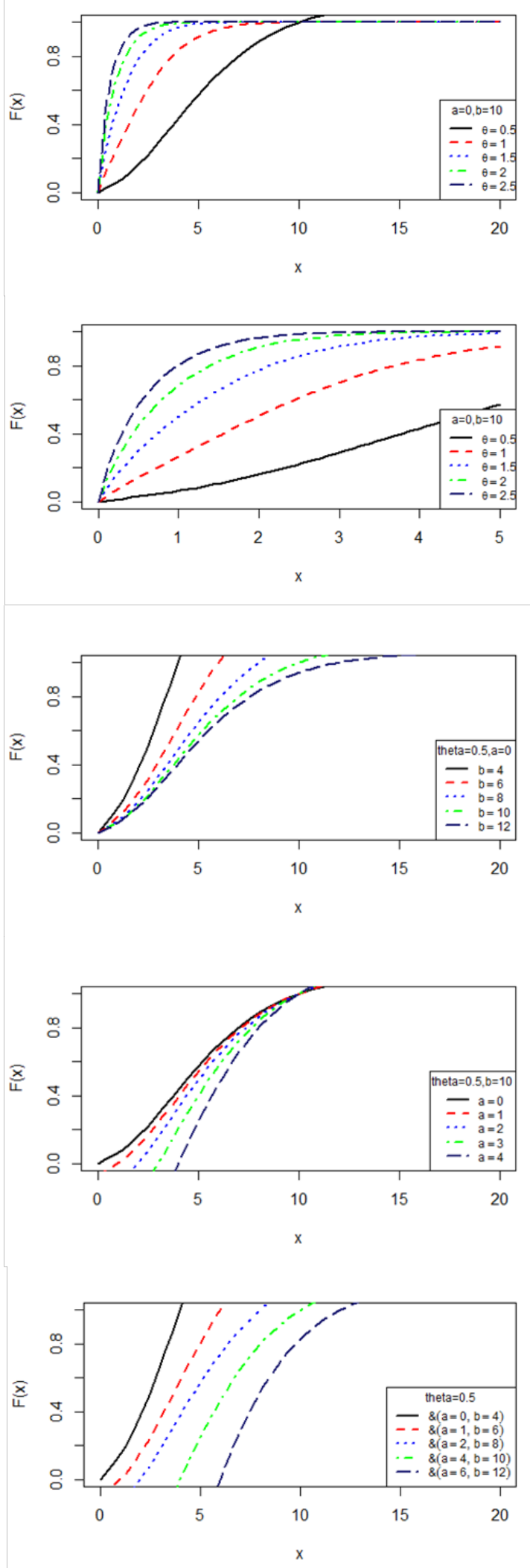

Figure $2 \mathrm{cdf}$ plots of TAD for varying values of parameter.

Definition 2: Let $X$ be random variable which is distributed as Truncated Akash distribution (TAD) with location parameters $a, b$ 
and scale $\theta$ and denoted by $\operatorname{TAD}(a, b, \theta)$. The pdf and cdf of $\mathrm{X}$ are respectively:

$$
\begin{aligned}
& f(x ; \theta)=\frac{\theta^{3}\left(x^{2}+1\right) e^{-\theta x}}{a \theta(a \theta+2) e^{-\theta a}-b \theta(b \theta+2) e^{-\theta b}+\left(\theta^{2}+2\right)\left(e^{-\theta a}-e^{-\theta b}\right)} \\
& F(x ; \theta)=\frac{a \theta(a \theta+2) e^{-\theta a}-x \theta(x \theta+2) e^{-\theta x}+\left(\theta^{2}+2\right)\left(e^{-\theta a}-e^{-\theta x}\right)}{a \theta(a \theta+2) e^{-\theta a}-b \theta(b \theta+2) e^{-\theta b}+\left(\theta^{2}+2\right)\left(e^{-\theta a}-e^{-\theta b}\right)}
\end{aligned}
$$

where $-\infty<a \leq x \leq b<\infty$, and $\theta>0$

Performance of pdf and cdf of TAD for varying values of parameters has been illustrated in the figure $1 \& 2$ respectively.

\section{Survival and hazard function}

The survival function $S(x)$ and the hazard function $h(x)$ of TAD are defined as

$$
\begin{aligned}
& S(x)=1-F(x)=\frac{x \theta(x \theta+2) e^{-\theta x}-b \theta(b \theta+2) e^{-\theta b}+\left(\theta^{2}+2\right)\left(e^{-\theta x}-e^{-\theta b}\right)}{a \theta(a \theta+2) e^{-\theta a}-b \theta(b \theta+2) e^{-\theta b}+\left(\theta^{2}+2\right)\left(e^{-\theta a}-e^{-\theta b}\right)} \\
& h(x)=\frac{f(x)}{S(x)}=\frac{\theta^{3}\left(x^{2}+1\right) e^{-\theta x}}{x \theta(x \theta+2) e^{-\theta x}-b \theta(b \theta+2) e^{-\theta b}+\left(\theta^{2}+2\right)\left(e^{-\theta x}-e^{-\theta b}\right)}
\end{aligned}
$$

It is obvious that $h(x)$ is independent from parameter $a$. Behavior of hazard function of TAD for varying values of parameter is presented in figure 3 .
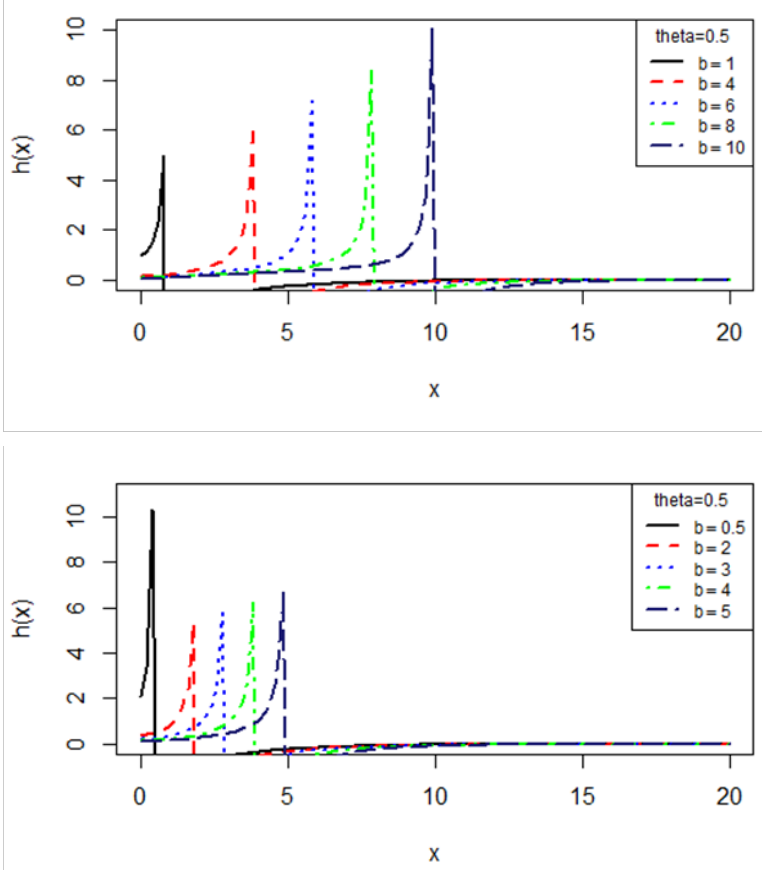

Figure $3 \mathrm{~h}(\mathrm{x})$ plots of TAD for varying values of parameter.

\section{Moments and Mathematical Properties}

Theorem: Suppose $X$ follows doubly TAD $(\theta, a, b)$. Then the $r$ th moment about origin $\mu_{r}^{\prime}$ of TAD is

$\mu_{r}^{\prime}=\frac{\theta^{2}\{\gamma(r+1, \theta b)-\gamma(r+1, \theta a)\}+\{\gamma(r+2, \theta b)-\gamma(r+2, \theta a)\}}{\theta^{r}\left(a \theta(a \theta+2) e^{-\theta a}-b \theta(b \theta+2) e^{-\theta b}+\left(\theta^{2}+2\right)\left(e^{-\theta a}-e^{-\theta b}\right)\right)} ; r=1,2,3, \ldots$
Proof: Considering

$$
K=\left\{a \theta(a \theta+2) e^{-\theta a}-b \theta(b \theta+2) e^{-\theta b}+\left(\theta^{2}+2\right)\left(e^{-\theta a}-e^{-\theta b}\right)\right\}
$$

in (1.6), we have

$$
\begin{gathered}
\mu_{r}^{\prime}=\frac{\theta^{3}}{K} \int_{a}^{b} x^{r}\left(1+x^{2}\right) e^{-\theta x} d x \\
=\frac{\theta^{3}}{K}\left[\int_{a}^{b} e^{-\theta x} x^{r} d x+\int_{a}^{b} e^{-\theta x} x^{r+2} d x\right]
\end{gathered}
$$

Taking $u=\theta x, x=\frac{u}{\theta}$

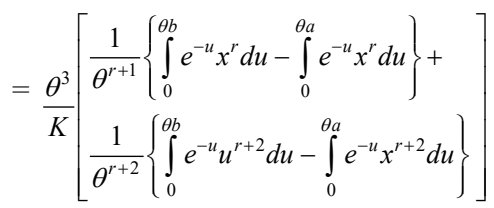

Where $\gamma(\alpha, z)=\int_{0}^{z} e^{-x} x^{\alpha-1} d x, \alpha>0, x>0$ is the lower incomplete gamma function

$$
\begin{array}{r}
=\frac{\theta^{3}}{K}\left[\frac{\gamma(r+1, \theta b)-\gamma(r+1, \theta a)}{\theta^{r+1}}+\frac{\gamma(r+3, \theta b)-\gamma(r+3, \theta a)}{\theta^{r+3}}\right] \\
=\frac{1}{K}\left[\frac{\theta^{2}\{\gamma(r+1, \theta b)-\gamma(r+1, \theta a)\}+\{\gamma(r+3, \theta b)-\gamma(r+3, \theta a)\}}{\theta^{r}}\right] \\
=\frac{\theta^{2}\{\gamma(r+1, \theta b)-\gamma(r+1, \theta a)\}+\{\gamma(r+2, \theta b)-\gamma(r+2, \theta a)\}}{\theta^{r}\left(a \theta(a \theta+2) e^{-\theta a}-b \theta(b \theta+2) e^{-\theta b}+\left(\theta^{2}+2\right)\left(e^{-\theta a}-e^{-\theta b}\right)\right)}
\end{array}
$$

Now putting $r=1,2$ in (4.1), mean and variance can be obtained as

$$
\begin{aligned}
& \mu_{1}^{\prime}=\frac{\theta^{2}\{\gamma(2, \theta b)-\gamma(2, \theta a)\}+\{\gamma(4, \theta b)-\gamma(4, \theta a)\}}{\theta\left(a \theta(a \theta+2) e^{-\theta a}-b \theta(b \theta+2) e^{-\theta b}+\left(\theta^{2}+2\right)\left(e^{-\theta a}-e^{-\theta b}\right)\right)} \\
& \mu_{2}^{\prime}=\frac{\theta^{2}\{\gamma(3, \theta b)-\gamma(3, \theta a)\}+\{\gamma(5, \theta b)-\gamma(5, \theta a)\}}{\theta^{2}\left(a \theta(a \theta+2) e^{-\theta a}-b \theta(b \theta+2) e^{-\theta b}+\left(\theta^{2}+2\right)\left(e^{-\theta a}-e^{-\theta b}\right)\right)} \\
& \text { Variance } \mu_{2}=\mu_{2}^{\prime}-\left(\mu_{1}^{\prime}\right)^{2}
\end{aligned}
$$

Similarly rest two moment of origin as well as coefficient of variation, coefficient of skewness, coefficient of kurtosis and Index of dispersion can be obtained, substituting $r=3,4$ in the equation (4.1), which are as follows:

$$
\begin{aligned}
& \mu_{3}^{\prime}=\frac{\theta^{4}\{\gamma(4, \theta b)-\gamma(4, \theta a)\}+\{\gamma(6, \theta b)-\gamma(6, \theta a)\}}{\theta^{3}\left(a \theta(a \theta+2) e^{-\theta a}-b \theta(b \theta+2) e^{-\theta b}+\left(\theta^{2}+2\right)\left(e^{-\theta a}-e^{-\theta b}\right)\right)} \\
& \mu_{4}^{\prime}=\frac{\theta^{4}\{\gamma(5, \theta b)-\gamma(5, \theta a)\}+\{\gamma(8, \theta b)-\gamma(8, \theta a)\}}{\theta^{4}\left(\begin{array}{l}
\left(a^{3} \theta^{3}+3 a^{2} \theta^{2}+6 a \theta+\theta^{4}+6\right) e^{-\theta a}- \\
\left(b^{3} \theta^{3}+3 b^{2} \theta^{2}+6 b \theta+\theta^{4}+6\right) e^{-\theta b}
\end{array}\right)}
\end{aligned}
$$

Coefficient of Variation $=\frac{\left(\mu_{2}^{\prime}-\left(\mu_{1}^{\prime}\right)^{2}\right)^{1 / 2}}{\mu_{1}^{\prime}}$, Coefficient of Skweness $=\frac{\left(\mu_{3}^{\prime}+3 \mu_{2}^{\prime} \mu_{1}^{\prime}-\left(\mu_{1}^{\prime}\right)^{2}\right)}{\left(\mu_{2}^{\prime}-\left(\mu_{1}^{\prime}\right)^{2}\right)^{3 / 2}}$, Coefficient of Kurtosis= 


$$
\frac{\left(\mu_{4}^{\prime}-4 \mu_{3}^{\prime} \mu_{1}^{\prime}+6 \mu_{2}^{\prime}\left(\mu_{1}^{\prime}\right)^{2}-3\left(\mu_{1}^{\prime}\right)^{4}\right)}{\left(\mu_{2}^{\prime}-\left(\mu_{1}^{\prime}\right)^{2}\right)^{2}},
$$
Index of dispersion $=\frac{\left(\mu_{2}^{\prime}-\left(\mu_{1}^{\prime}\right)^{2}\right)}{\mu_{1}^{\prime}}$, However, they can be easily
obtained.

\section{Maximum Likelihood Method Estimation}

Let $\left(x_{1}, x_{2}, x_{3}, \ldots, x_{n}\right)$ be a random sample of size $n$ from (1.1). The likelihood function, $L$ of TAD is given by

$$
L=\left(\frac{\theta^{3}}{a \theta(a \theta+2) e^{-\theta a}-b \theta(b \theta+2) e^{-\theta b}+\left(\theta^{2}+2\right)\left(e^{-\theta a}-e^{-\theta b}\right)}\right)^{n} \prod_{i=1}^{n}\left(1+x_{i}^{2}\right) e^{-n \theta \bar{x}}
$$

The $\log$ likelihood function is thus obtained as

$$
\ln L=n \ln \left(\frac{\theta^{3}}{a \theta(a \theta+2) e^{-\theta a}-b \theta(b \theta+2) e^{-\theta b}+\left(\theta^{2}+2\right)\left(e^{-\theta a}-e^{-\theta b}\right)}\right)+\sum_{i=1}^{n} \ln \left(1+x_{i}^{2}\right)-n \theta \bar{x}
$$

Taking $\hat{a}=\min \left(x_{1}, x_{2}, x_{3}, . ., x_{n}\right), \hat{b}=\max \left(x_{1}, x_{2}, x_{3}, \ldots, x_{n}\right)$, the maximum likelihood estimate $\hat{\theta}$ of parameter $\theta$ is the solution of the $\log$-likelihood equation $\frac{\partial \log L}{\partial \theta}=0$. It is obvious that $\frac{\partial \log L}{\partial \theta}=0$ will not be in closed form and hence some numerical optimization technique can be used e the equation for $\theta$. In this paper the nonlinear method available in R software has been used to find the MLE of the parameter $\theta$.

\section{Applications on Life time data}

In this section, TAD has been applied to three datasets using maximum likelihood estimates. Parameter $\theta$ is estimated whereas another parameters $\mathrm{a}$, and $\mathrm{b}$ are considered as lowest and highest values of data. i. e. $\hat{a}=\min \left(x_{1}, x_{2}, x_{3}, . ., x_{n}\right)$ and $\hat{b}=\max \left(x_{1}, x_{2}, x_{3}, \ldots, x_{n}\right)$. Goodness of fit has been decided using Akaike information criteria (AIC), Bayesian Information criteria (BIC) and Kolmogorov Simonov test (KS) values respectively, which are calculated for each distribution and also compared with p-value and given in the table 1,2 \&3. As we know that best goodness of fit of the distribution can be decided on the basis of minimum value of KS, AIC and BIC.

Table I MLE's, Standard Errors, - 2ln L,AIC, BIC, K-S and p-values of the fitted distributions for data set-5

\begin{tabular}{llllllll}
\hline Distributions & ML Estimates & Standard Errors & $-2 \ln L$ & AIC & BIC & K-S & p-value \\
\hline TAD & $\hat{\theta}=0.03917$ & $\mathbf{0 . 0 0 3 0 3}$ & $\mathbf{9 3 9 . 1 3}$ & $\mathbf{9 4 1 . 1 3}$ & $\mathbf{9 4 2 . 0 5}$ & $\mathbf{0 . 1 5 3}$ & $\mathbf{0 . 0 1 7}$ \\
TLD & $\hat{\theta}=0.02199$ & 0.00273 & 958.88 & 960.88 & 962.31 & 0.186 & 0.001 \\
Akash & $\hat{\theta}=0.04387$ & 0.00253 & 950.97 & 952.97 & 954.40 & 0.194 & 0.001 \\
Ishita & $\hat{\theta}=0.04390$ & 0.002533 & 950.92 & 9952.92 & 954.35 & 0.194 & 0.001 \\
Lindley & $\hat{\theta}=0.02886$ & 0.002038 & 983.10 & 985.10 & 986.54 & 0.252 & 0.000 \\
Exponential & $\hat{\theta}=0.01463$ & 0.001457 & 1044.87 & 1046.87 & 1048.30 & 0.336 & 0.000 \\
\hline
\end{tabular}

Table 2 MLE's, Standard Errors, - 2ln L,AIC, BIC, K-S and p-values of the fitted distributions for data set-2

\begin{tabular}{|c|c|c|c|c|c|c|c|}
\hline Distributions & ML Estimates & Standard Errors & $-2 \ln L$ & AIC & BIC & K-S & p-value \\
\hline TAD & $\hat{\theta}=0.70314$ & $0.1867 \mid$ & I | 0.76 & 112.76 & 114.68 & 0.152 & 0.079 \\
\hline TLD & $\hat{\theta}=0.28986$ & 0.184873 & 112.19 & 114.19 & 115.63 & 0.157 & 0.065 \\
\hline Akash & $\hat{\theta}=0.96472$ & 0.06460 & 224.27 & 226.27 & 227.27 & 0.362 & 0.000 \\
\hline Ishita & $\hat{\theta}=0.93156$ & 0.05602 & 223.14 & 225.14 & 226.13 & 0.330 & 0.000 \\
\hline Lindley & $\hat{\theta}=0.65450$ & 0.05803 & 238.38 & 240.38 & 241.37 & 0.401 & 0.000 \\
\hline Exponential & $\hat{\theta}=0.40794$ & 0.04911 & 261.73 & 263.73 & 264.73 & 0.448 & 0.000 \\
\hline
\end{tabular}

\begin{tabular}{llllllll}
\hline Distributions & ML Estimates & Standard Errors & $-2 \ln L$ & AIC & BIC & K-S & p-value \\
\hline TAD & $\hat{\theta}=0.08776$ & $\mathbf{0 . 0 2 4 2 4 I}$ & $\mathbf{2 0 1 . 9 6}$ & $\mathbf{2 0 3 . 9 6}$ & $\mathbf{2 0 5 . 5 8}$ & $\mathbf{0 . 1 1 2}$ & $\mathbf{0 . 7 8 6}$ \\
TLD & $\hat{\theta}=0.05392$ & 0.023917 & 202.18 & 204.18 & $205.6 \mathrm{I}$ & 0.117 & 0.738 \\
Akash & $\hat{\theta}=0.09706$ & 0.01004 & 240.68 & 242.68 & 242.67 & 0.298 & 0.005 \\
Ishita & $\hat{\theta}=0.097328$ & 0.01008 & 240.48 & 242.48 & 243.48 & 0.297 & 0.006 \\
Lindley & $\hat{\theta}=0.06299$ & 0.00800 & 253.98 & 255.98 & 256.98 & 0.365 & 0.000 \\
Exponential & $\hat{\theta}=0.032452$ & 0.00582 & 274.52 & 276.52 & 277.52 & 0.458 & 0.000 \\
\hline
\end{tabular}

Table 3 MLE's, Standard Errors, - 2In L,AIC, BIC, K-S and p-values of the fitted distributions for data set-3 
Data Set 1: The data is given by Birnbaum and Saunders ${ }^{11}$ on the fatigue life of $6061-\mathrm{T} 6$ aluminum coupons cut parallel to the direction of rolling and oscillated at 18 cycles per second. The data set consists of 100 observations with maximum stress per cycle 31,000 psi. The data ( $\times 10^{-3}$ ) are presented below (after subtracting 65 ).

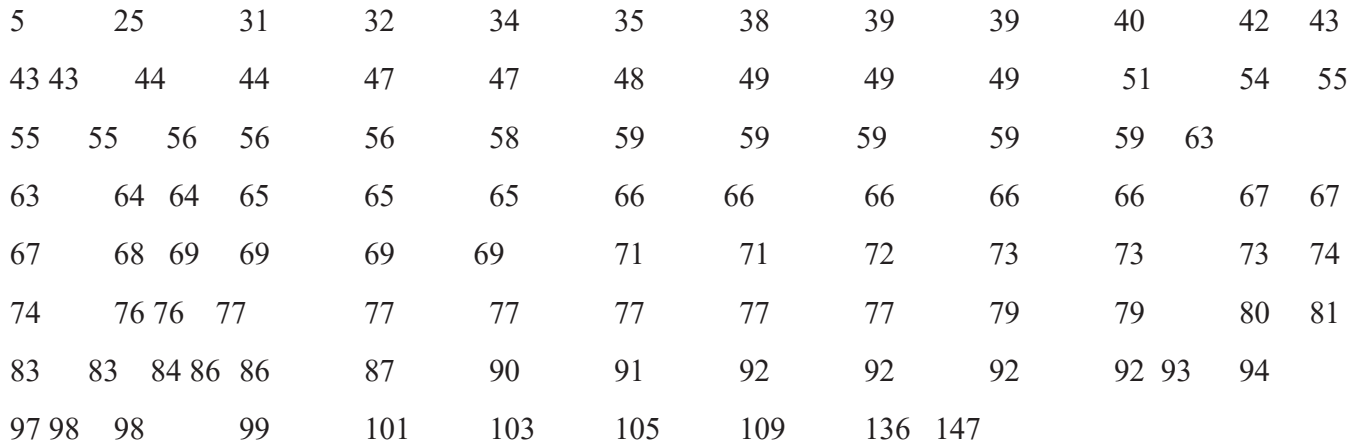

Data Set 2: This data set is the strength data of glass of the aircraft window reported by Fuller, Frieman, Quinn, Quinn, and Carter: ${ }^{12}$

$\begin{array}{lllllllllllll}18.83 & 20.8 & 21.657 & 23.03 & 23.23 & 24.05 & 24.321 & 25.5 & 25.52 & 25.8 & 26.69 & 26.77 & \\ 26.78 & 27.05 & 27.67 & 29.9 & 31.11 & 33.2 & 33.73 & 33.76 & 33.89 & 34.76 & 35.75 & 35.9136 .98 & 37.0837 .09 \\ 39.58 & 44.045 & 45.29 & 45.381 & & & & & & & & & \end{array}$

Data Set 3: The following data represent the tensile strength, measured in GPa, of 69 carbon fibers tested under tension at gauge lengths of $20 \mathrm{~mm}$, Bader and Priest: ${ }^{13}$

$\begin{array}{llllllllllllll} & 1.312 & 1.314 & 1.479 & 1.552 & 1.700 & 1.803 & 1.861 & 1.865 & 1.944 & 1.958 & 1.966 & 1.997 & \\ & 2.006 & 2.021 & 2.027 & 2.055 & 2.063 & 2.098 & 2.140 & 2.179 & 2.224 & 2.240 & 2.253 & 2.270 & 2.272 \\ 2.274 & 2.301 & 2.301 & 2.359 & 2.382 & 2.382 & 2.426 & 2.434 & 2.435 & 2.478 & 2.490 & 2.511 & 2.514 & 2.535 \\ 2.554 & 2.566 & 2.570 & 2.586 & 2.629 & 2.633 & 2.642 & 2.648 & 2.684 & 2.697 & 2.726 & 2.770 & 2.773 & 2.80 \\ 2.809 & 2.818 & 2.821 & 2.848 & 2.880 & 2.954 & 3.012 & 3.067 & 3.084 & 3.090 & 3.096 & 3.128 & 3.233 & 3.433\end{array}$

3.5853 .858

Fitted plots of the considered distributions are presented in Figure 4, 5 and 6, respectively.

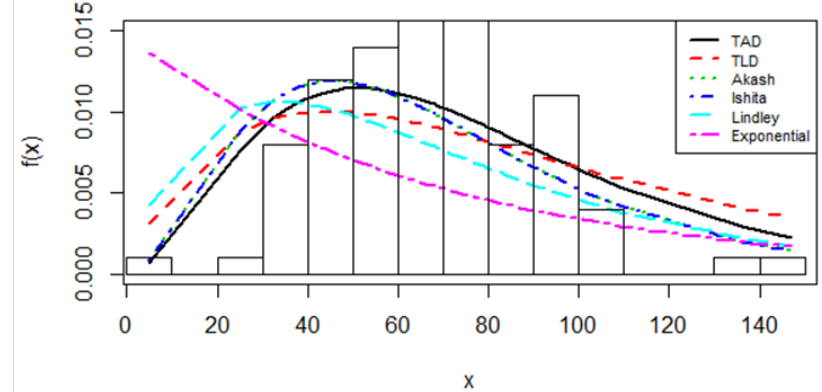

Figure 4 Fitted plots of distributions for the dataset-I.

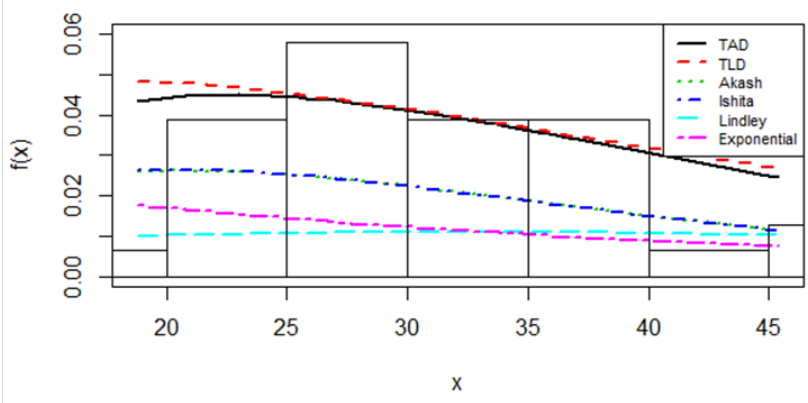

Figure 5 Fitted plot of distributions for data set-2.

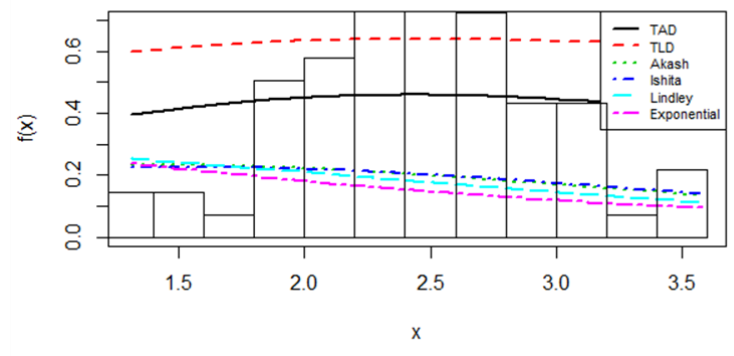

Figure 6 Fitted plot of distribution for data set-3.

\section{Conclusion}

In this paper, truncated Akash distribution (TAD) has been proposed. Its statistical properties including survival function and hazard rate have been discussed. Its moments including Coefficient of variation, Skewness, Kurtosis and Index of dispersion have derived. Maximum likelihood method has been used for estimation of its parameter. Goodness of fit of TAD has been discussed with three life time datasets and compared with truncated Lindley, Akash, Ishita, Lindley and exponential distributions. It has been observed that TAD gives good fit over TLD (truncated Lindley Distribution), Akash, Ishita, Lindley and exponential distribution.

\section{References}

1. Lindley DV. Fiducial distributions and Bayes' Theorem. Journal of the Royal Statistical Society, Series B. 1958;20(1):102-107. 
2. Ghitany ME, Atieh B, Nadarajah S. Lindley distribution and its Applications. Mathematics Computing and Simulation. 2008;78(4):493506.

3. Shanker R. Akash distribution and its application. International Journal of Probability and Statistics. 2015;4(3):65-75.

4. Shanker R, Shukla KK. Ishita distribution and its applications. Biometrics \& Biostatistics International Journal. 2017;5:1-9.

5. Shukla KK. Pranav distribution with Properties and Applications Biometrics and Biostatistics International Journal. 2018;7(3):244-254.

6. Johnson NL, Kotz S, Balakrishnan N. Continuous univariate distributions. New York, USA: Wiley; 1994. p. 784.

7. Zange Z, Xie M. On the upper truncated Weibull distribution and its reliability implications. Reliability Engineering \& System Safety. 2011;96(1):194-200.

8. Aryuyuen S, Bodhisuwan W. The truncated power Lomax distribution: Properties and applications. Walailak Journal of Science and Technology. 2018;16:655-668.
9. Zaninetti L, Ferraro M. On the truncated Pareto distribution with applications. Central European Journal of Physics. 2008;6:1-6.

10. Singh SK, Singh U, Sharma VK. The truncated Lindley distribution: Inference and application. Journal of Statistics Applications \& Probability. 2014;3(2):219-228.

11. Birnbaum ZW, Saunders SC. Estimation for a family of life distribution with applications to fatigue. Journal of Applied Probability. 1969; 6(2):328-347.

12. Fuller EJ, Frieman S, Quinn J, et al. Fracture mechanics approach to the design of glass aircraft windows: A case study. SPIE Proc. 1994;2286:419-430.

13. Bader MG, Hriest AM. Statistical aspects of fiber and bundle strength in hybrid composites. In: Hayashi T, Kawata K, Umekawa S, editors. Progressin Science in Engineering Composites. Tokyo, Japan: ICCMIV. 1982;1129-1136. 